\title{
A novel Tn antigen epitope-recognizing antibody for MUC1 predicts clinical outcome in patients with primary lung adenocarcinoma
}

\author{
TATSUYA KATO $^{1}$, HIDEKI UJIIE ${ }^{1}$, KANAKO C. HATANAKA ${ }^{2}$, AYAE NANGE ${ }^{3}$, ASAMI OKUMURA ${ }^{3}$, \\ KAHO TSUBAME $^{4}$, KENTATO NARUCHI ${ }^{5}$, MASAHARU SATO ${ }^{5}$, KICHIZO KAGA ${ }^{1}$, \\ YOSHIHIRO MATSUNO ${ }^{6}$, SATORU WAKASA ${ }^{1}$ and YUTAKA HATANAKA ${ }^{3}$

\footnotetext{
${ }^{1}$ Department of Cardiovascular and Thoracic Surgery, Hokkaido University Faculty and School of Medicine, Sapporo, Hokkaido 060-8638; ${ }^{2}$ Clinical Research and Medical Innovation Center, Hokkaido University Hospital; ${ }^{3}$ Research Division of Companion Diagnostics, Hokkaido University Hospital, Sapporo, Hokkaido, 060-8648; ${ }^{4}$ Department of Pathology, Hokkaido University Graduate School of Medicine, Sapporo, Hokkaido 060-8638;

${ }^{5}$ Medicinal Chemistry Pharmaceuticals, Co., Ltd., Sapporo, Hokkaido 060-0009;

${ }^{6}$ Department of Surgical Pathology, Hokkaido University Hospital, Sapporo, Hokkaido 060-8648, Japan
}

Received July 14, 2020; Accepted November 30, 2020

DOI: $10.3892 / 01.2021 .12463$

\begin{abstract}
Mucin 1 (MUC1) expression is upregulated in multiple types of cancer, including lung cancer. However, the conventional anti-MUC1 antibody is not useful for the differentiation of malignant lung tumors and benign lesions due to its limited specificity. Our previous study screened a novel epitope-defined antibody against cancer-associated sugar chain structures that specifically recognizes the MUC1 Tn antigen (MUC1-Tn ED Ab). In the present study, its potential utility as a diagnostic marker and therapeutic tool for lung adenocarcinoma (ADC) was examined. Immunohistochemical analysis of a lung ADC tissue microarray was performed using the MUC1-Tn ED Ab (clone SN-102), and the results were compared with those of another clone and commercially available MUC1 antibodies. The association between positive immunoreactivity of SN-102 and clinicopathologic factors was analyzed. Furthermore, the association between MUC1-Tn expression and epithelial-mesenchymal transition markers and radiological characteristics was analyzed. Moderate or high MUC1-Tn expression (MUC1-Tn-H) was observed in $138(78.9 \%)$ of the 175 lung ADC cases. MUC1-Tn-H was associated with male sex, cigarette smoking, tumor extension, pleural invasion, and higher preoperative serum
\end{abstract}

Correspondence to: Dr Tatsuya Kato, Department of Cardiovascular and Thoracic Surgery, Hokkaido University Faculty and School of Medicine, N15W7, Kita-ku, Sapporo, Hokkaido 060-8638, Japan E-mail:katotatu7@msn.com

Key words: mucin 1, Tn antigen, epitope-defined antibody, epithelial-mesenchymal transition, prognostic factor, lung cancer carcinoembryonic antigen and cytokeratin 19 fragment levels. Tumors with MUC1-Tn-H had higher consolidation/tumor ratios according to computed tomography and greater uptakes of ${ }^{18} \mathrm{~F}$-fluorodeoxyglucose. A total of $46(26.9 \%)$ of the tumors had mesenchymal features, and MUC1-Tn positivity was higher in the mesenchymal group than in the epithelial and intermediate groups $(\mathrm{P}<0.01$ and $\mathrm{P}<0.01$, respectively). Patients with tumors exhibiting MUC1-Tn-H had significantly shorter 5-year overall and disease-free survival times $(\mathrm{P}=0.011$ and $\mathrm{P}<0.001$, respectively). Additionally, MUC1-Tn-H was identified as an independent prognostic factor in multivariate analysis $(\mathrm{P}=0.024)$. MUC1-Tn is specific for lung cancer cells and can improve diagnostic capabilities. Additionally, it may be a potential therapeutic target in lung ADC.

\section{Introduction}

Lung cancer is the leading cause of cancer-related death worldwide, and the 5-year survival of patients with advanced-stage disease is poor (1). Currently, serological diagnosis for primary lung cancer is poor, even when a combination of several serum tumor markers is used. Additionally, there are few reliable tumor markers to detect recurrence postoperatively. The lack of major improvements in the diagnostic and survival rates for lung cancer has driven a search for novel markers aimed at improving early detection of primary lung cancer and recurrence after surgery using a high-specificity antibody.

MUC1 (mucin 1, cell surface associated; CD227) is a high molecular-weight transmembrane glycoprotein (2). The MUC1 N-terminal subunit (MUC1-N) contains highly glycosylated tandem repeats (TRs) that are a physical characteristic of the mucin family (3). A TR consists of a constant amino acid sequence rich in serine (Ser) and threonine (Thr) residues $(4,5)$. MUC1-N forms a complex with the MUC1 
C-terminal subunit at the cell membrane and is shed from the surface of cancer cells, leading to increased plasma levels of MUC1. Although mucins, including MUC1, are also highly expressed in healthy epithelial cells, the sugar chain structure of MUC1 is completely different between normal and tumor cells. Normal cells have modifications in long-branched sugar chains, whereas cancer cells express various simple and short sugar chain antigens called O-glycans (e.g., Tn, sialyl-Tn and sialyl-Lewis-X). Abnormally glycosylated mucins in malignant cells are currently being researched due to unique post-translational modification of mucin backbones by carbohydrates (6). O-glycosylation is initiated by the polypeptide $\mathrm{N}$-acetylgalactosaminyltransferase, which utilizes UDP-GalNAc to add GalNAc to Ser/Thr residues. The addition of GalNAc to Ser/Thr via $\alpha$-linkage forms the Tn antigen (GalNAc $\alpha 1-O-S e r / T h r, C D 175)$ (7). Increased expression of Tn has been attributed to inactive T-synthase, core 1 synthase glycoprotein- $\mathrm{N}$-acetylgalactosamine, and 3- $\beta$-galactosyltransferase (C1GALT1) (8). Aberrant O-glycosylation due to disruption of C1GALT1 contributes to progression and metastasis of pancreatic cancer in mice (9). Additionally, C1GALT1 unusually requires a private chaperone, core $1 \beta 3$-Gal-T-specific molecular chaperone (COSMC), for folding and activity. Loss of COSMC due to somatic mutations or hypermethylation also causes the dysregulation of $\mathrm{O}$-glycans and induces traditional oncogenic features, including hyperproliferation, loss of tissue architecture and disruption of basement membrane adhesion, and invasive growth of cancer (10).

It is important to understand the recognition site of MUC1 antibody. The conventional MUC1 antibody recognizes amino acids in the TR domain or a carbohydrate epitope of the MUC1 protein, and this lack of specificity leads to diametrically opposite outcomes in lung cancer prognosis analysis (11-13). Krebs von den Lungen-6 (KL-6), an important biomarker of interstitial lung diseases (ILD), is now classified as a MUC1 protein. Regenerating type II pneumocytes are the primary cellular source of KL-6/MUC1 in the lungs of patients with ILD. KL-6/MUC1 is detectable in the sera of $70-100 \%$ of patients with various ILD (14); however, it is also detected in the sera of lung cancer patients (15), raising the false-positive rate of lung cancer diagnosis. Further, an antibody that can selectively recognize this cancer-specific change in sugar chain structure would be useful for early diagnosis of lung cancer. Although significant research on MUC1 in lung cancer has been conducted, research evidence about the importance of MUC1-Tn as a diagnostic and prognostic marker is lacking. Therefore, we screened a high-specificity monoclonal antibody against MUC1 TR glycopeptides bearing the Tn antigen (MUC1-Tn antigen epitope-defined antibody [MUC1-Tn ED $\mathrm{Ab]}$ ) through an innovative technique utilizing the production of fine glycopeptides and a screening system for a specific antibody using epitope analyses (16).

We aimed to examine the expression of the MUC1-Tn antigen in primary lung adenocarcinoma (ADC) and assessed relationships between its expression and clinical impact on prognosis by an immunohistochemical (IHC) study with paraffin-embedded tissue microarray (TMA) sections. This could lead to the development of MUC1-Tn as a novel high-specificity diagnostic marker and therapeutic target for lung ADC.

\section{Materials and methods}

Clinical lung cancer tissue samples. A total of 175 lung ADC tissue samples were obtained from patients who underwent surgery at the Hokkaido University Hospital with the patients' informed consent. Detailed clinical and pathological information was collected retrospectively for all patients. The median follow-up period was 66.9 months for patients who were alive. The median age of the patients at the time of diagnosis was 68 years (interquartile range, $60-73$ years), and $52.0 \%$ of the patients were women (Table I). Histological diagnoses of tumors were based on the 2015 World Health Organization Classification (17). All tumors were staged according to the pathological tumor/node/metastasis classification (8th edition) of the Union for International Cancer Control (18). All tumors were histologically reviewed by an experienced pathologist (K.C.H.).

Tissue microarray construction and immunohistochemistry. Tissue areas were selected for sampling based on visual alignment with the corresponding hematoxylin and eosin-stained sections on slides. TMA blocks were then constructed using a manual tissue microarrayer (JF-4; Sakura Finetek Japan) with a 1.5-mm diameter needle. Formalin-fixed paraffin-embedded specimens were cut into $4-\mu \mathrm{m}$-thick sections, dewaxed with xylenes, and rehydrated through a graded ethanol series. The IHC protocol for MUC1, MUC1-Tn, Vimentin, and E-cadherin was as follows: i) for antigen retrieval, sections were treated with Target Retrieval Solution, Low pH (K8005; Dako) for Vimentin and High pH (EnVision FLEX Mini Kit; Dako) for all other antibodies at $97^{\circ} \mathrm{C}$ for $20 \mathrm{~min}$ before inhibiting endogenous peroxidase activity for $5 \mathrm{~min}$ at room temperature (RT) with EnVision FLEX Peroxidase-Blocking Reagent (Dako); ii) sections were incubated with clone SN-102 or SN-110 (1:500 and 1:100, respectively), commercially available MUC1 antibodies (Ma552, 1:100; Novocastra and Ma695, 1:1600; Biocare Medical), anti-E-cadherin monoclonal antibody (M3612, clone NCH-38, 1:50; Dako), or anti-Vimentin monoclonal antibody (IR630, clone V9, Ready-to-use; Dako) at the final concentration using mixed antibody diluent (S2022; Dako) for $30 \mathrm{~min}$ at RT; iii) a polymer-based detection with 3',3-Diaminobenzidine (DAB) system was used (DAB and DAB plus Chromogen Solution, Dako); and iv) sections were counterstained with hematoxylin. Slides were dehydrated and placed on coverslips.

Evaluation of immunohistochemical staining. Digital images of IHC-stained TMA slides were obtained at $\mathrm{x} 4-\mathrm{x} 20$ magnification using a whole slide scanner (NanoZoomer 2.0-HT slide scanner; Hamamatsu Photonics). Annotation of tumor regions on slides was performed by researchers (T.K and H.U.) blinded to the clinical follow-up data using Aperio's annotation software (ImageScope Viewing Software: Positive Pixel Count v9.1, Aperio ImageScope ${ }^{\circledR}$; Leica Microsystems Inc.). The weighted intensity of staining was graded as follows: grade 0 (negative), 1+ (WEAK positive: Intensity Threshold WEAK [Upper Limit $]=220$, [lower limit $]=175), 2+($ MEDIUM: [upper] $=175$, [lower] $=100)$, and 3+ (STRONG: [upper] $=100$, [lower] $=0)$ by default. The staining of SN-102 was quantified by IHC positivity, which was calculated as the number of positive pixels 
stained at each intensity level divided by the total number of pixels (the number of positive and negative pixels). According to the IHC positivity (Pos.), samples were finally divided into three groups based on MUC1-Tn expression (the threshold leading to the 1st $[\mathrm{Q} 1=0.04]$ and 3rd quartiles [Q3=0.14]): low MUC1-Tn expression was less than Q1 (MUC1-Tn-L, with a Pos. <0.04), moderate MUC1-Tn expression was between Q1 and Q3 (MUC1-Tn-M, with 0.04 a Pos. <0.14), and high MUC1-Tn expression was Q3 or greater (MUC1-Tn-H, with a Pos. $\geq 0.14$ ).

The levels of E-cadherin and Vimentin staining were independently evaluated by two investigators (T.K. and H.U.) and supervised by an experienced pathologist (K.C.H.). Cancer cells showing membrane and cytoplasmic staining for E-cadherin and those showing cytoplasmic staining for Vimentin were considered positive. The expression of E-cadherin was scored according to proportion and intensity scores using the following criteria: i) proportion of stained tumor cells was scored as $0(0 \%),+1(1-4 \%)$, $+2(5-49 \%)$, and $+3(\geq 50 \%)$; and ii) staining intensity was scored as +1 (weak), +2 (moderate), and +3 (strong). The scores were then multiplied to get a final E-cadherin staining score; final scores $\geq 9$ were considered positive. The expression of Vimentin was evaluated according to the proportion of Vimentin-positive cells $(\geq+2)$. According to these results, we classified tumor samples into three groups: epithelial (E-cadherin[+]/Vimentin[-]), intermediate (E-cadherin[+]/Vimentin[+]) or (E-cadherin[-]/Vimentin[-]), and mesenchymal (E-cadherin[-]/Vimentin[+]).

Statistical analysis. MUC1-Tn immunoreactivity was assessed for association with clinicopathologic variables using the $\chi^{2}$ test and Mann-Whitney U test for variables. Multiple comparison analyses (Kruskal-Wallis test followed by Dunn's test) were used to determine statistical significance for three groups. Receiver operating characteristic (ROC) curves were drawn using the data of MUC1-Tn positivity between lung cancer and normal lung, and the area under the ROC curve (AUC) was calculated. The Kaplan-Meier method was used to generate survival curves based on the status of MUC1-Tn expression, and survival differences were analyzed using the log-rank test. The primary end point was overall survival as measured from the date of surgery to the time of death. Disease-free survival was the period from surgery until the date of disease relapse. Univariate and multivariate analyses were performed using Cox proportional-hazards regression model. After crude analysis, we adjusted for pathologic variables (pT and $\mathrm{pN}$ ) in the multivariate analysis. We used normal ranges for tumor markers as cutoff values (carcinoembryonic antigen [CEA], 0-5.0 ng/ml and cytokeratin 19 fragment [CYFRA21-1], 0-2.1 ng/ml) in hazard analyses. The consolidation/tumor $(\mathrm{C} / \mathrm{T})$ ratio of the computed tomography $(\mathrm{CT})$ scan was dichotomized at its 1 st quartile value of 0.5 because the median was 1.0 in uni- and multivariate analyses using Cox's proportional hazards model. The maximum standardized uptake value $\left(\mathrm{SUV}_{\max }\right)$ of ${ }^{18} \mathrm{~F}$-fluorodeoxyglucose positron emission tomography $\left({ }^{18} \mathrm{~F}-\mathrm{FDG}-\mathrm{PET}\right)$ was dichotomized at its median value of 2.4. $\mathrm{P}<0.05$ was considered statistically significant. All analyses were performed using StatFlex version 6.0 for Windows (Artech).
Table I. Patient and tumor characteristics $(n=175)$.

\begin{tabular}{lc}
\hline Characteristic & Value \\
\hline Age (years), median (IQR) & $68(60,73)$ \\
Female, $\mathrm{n}(\%)$ & $91(52.0)$ \\
Tumor size (mm), median (IQR) & $22.0(15.0,30.0)$ \\
p-Stage ${ }^{a}, \mathrm{n}$ & \\
IA1-3 (p-T1a-cN0) & 99 \\
IB (p-T2aN0) & 29 \\
IIA (p-T2bN0) & 7 \\
IIB (p-T3N0/ p-T1a-cN1/p-T2a-bN1) & 17 \\
IIIA (p-T1a-cN2/p-T2a-bN2) & 1 \\
IIIB (p-T3N2/p-T4N2) & $4.0(2.7,8.0)$ \\
Preoperative serum CEA (ng/ml), & \\
median (IQR) & $1.3(1.0-2.2)$ \\
Preoperative serum CYFRA21-1 (ng/ml), & \\
median (IQR) & $1.0(0.5,1.0)$ \\
Consolidation/tumor ratio, median (IQR) & $2.4(1.1-5.0)$ \\
${ }^{18}$ F-FDG-PET SUV ${ }_{\text {max }}$, median (IQR) & \\
\hline
\end{tabular}

${ }^{a}$ TNM classification system of the Union for InternationalCancerControl (8th edition) (18). CEA, carcinoembryonic antigen; CYFRA21-1, cytokeratin 19 fragment; ${ }^{18} \mathrm{~F}$-FDG-PET, ${ }^{18} \mathrm{~F}$-fluorodeoxyglucose positron emission tomography; IQR, interquartile range; $\mathrm{SUV}_{\max }$, maximum standardized uptake value.

\section{Results}

Specificity and staining pattern of MUC1-Tn in normal lung and lung ADC tissues. First, we examined the specificity of commercially available MUC1 antibodies and antibodies that specifically recognize MUC1 with cancer-associated sugar chain structures using TMAs containing 20 normal lung tissue slides. Although commercially available monoclonal antibodies against MUC1 (Ma552 and Ma695) and the other MUC1-Tn ED antibody (clone SN-110) showed weak to moderate staining in normal lung tissue, the SN-102 antibody did not stain normal lung at all (Fig. 1A), suggesting that antibodies (except SN-102) may be difficult to use as therapeutic tools for lung cancer. Immunogen for SN-102 is shown in Fig. 1B. Although SN-102 recognizes the GalNAc region, its precise binding site remains unknown. The ROC curves and the AUC value for each antibody are shown in Fig. 1C, illustrating a high AUC value (0.95) for SN-102. Therefore, we performed subsequent analyses using SN-102. There was little staining in lung ADC tumor tissues using Ma552 and Ma695; however, a high level of staining was seen using SN-102, even in the same lung cancer TMA slides (Fig. 2A). Thus, different expression patterns were observed when MUC1-Tn and commercially available MUC1 antibodies were used. A representative overall view of a TMA slide is shown in Fig. 2B, illustrating high specificity of SN-102.

MUC1-Tn expression and correlation to clinicopathological parameters. Positive MUC1-Tn staining of tumor cells generally showed a membranous and cytoplasmic pattern in 


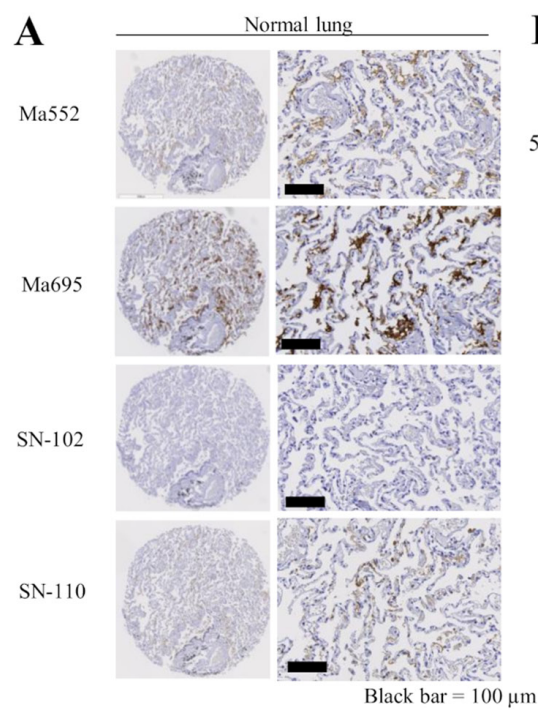

B
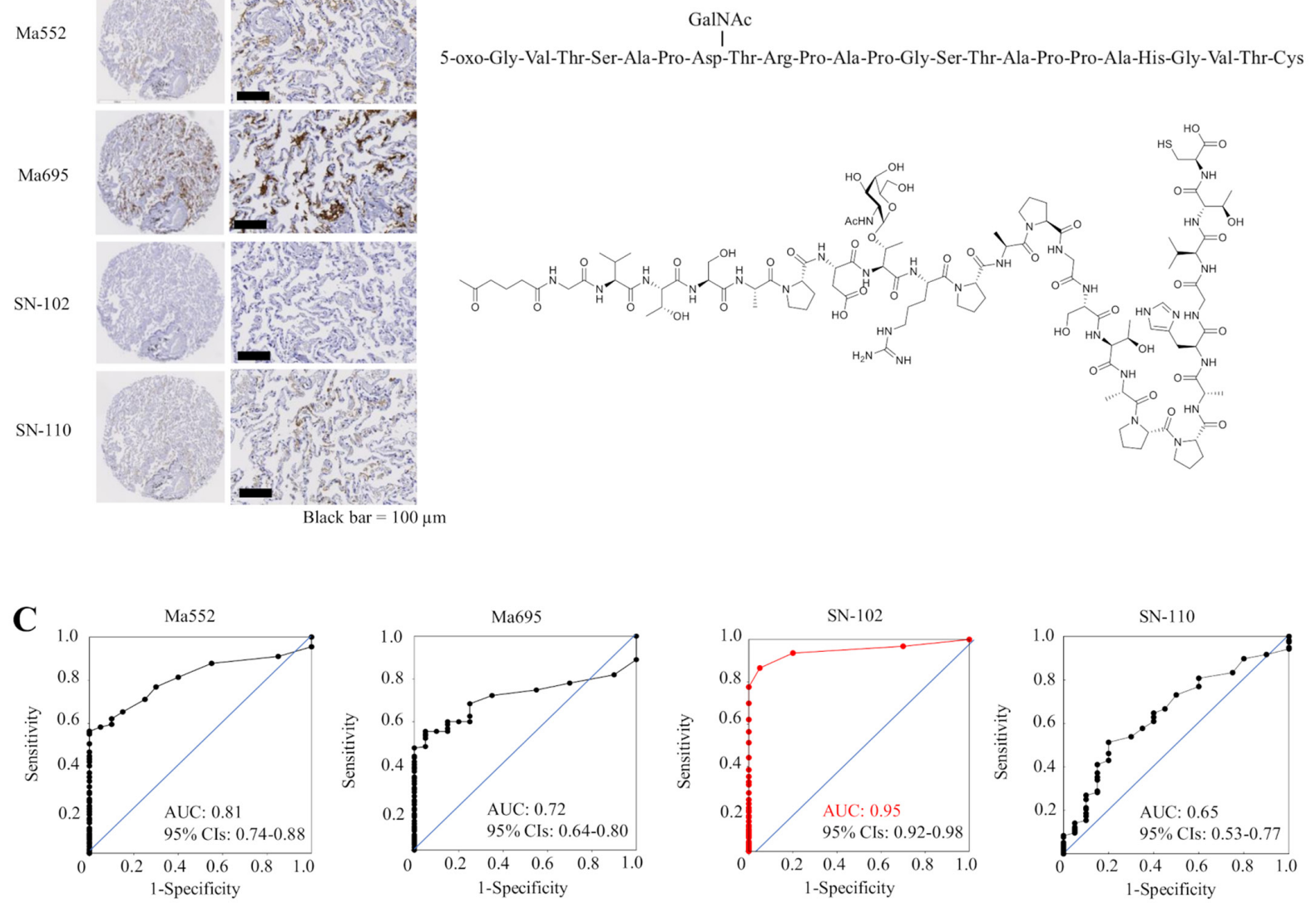

Figure 1. Expression levels of MUC1-Tn in normal lung tissue and the specificity of MUC1-Tn antibody. (A) No MUC1-Tn staining was observed in normal lung tissue when clone SN-102 was used. Commercially available MUC1 antibodies (Ma695 and Ma552) and clone SN-110 showed moderate expression in the alveolar epithelial cells. Scale bars, $100 \mu \mathrm{m}$. (B) Immunogen and its chemical formula for the SN-102 antibody. (C) ROC curves using the data of MUC1-Tn positivity between areas with lung cancer and those without and the AUC for each antibody. CIs, confidence intervals; MUC1-Tn, mucin 1 Tn-antigen; ROC, receiver operating characteristics; AUC, area under the curve.

cancer tissue (Fig. 2C). Of the 175 lung ADC cases examined, MUC1-Tn-L and MUC1-Tn-M were observed in $37(21.1 \%)$ and 94 cases (53.7\%), respectively. MUC1-Tn-H was observed in 44 cases $(25.1 \%)$ and was significantly associated with male sex $(\mathrm{P}=0.016)$, cigarette smoking $(\mathrm{P}=0.005)$, pT status $(\mathrm{P}<0.001)$, pleural invasion $(\mathrm{P}=0.008)$, lack of lymphatic and vascular invasion $(\mathrm{P}<0.001$ and $\mathrm{P}=0.005$, respectively), high preoperative serum CEA levels $(\mathrm{P}=0.010)$, and high preoperative serum CYFRA21-1 levels $(\mathrm{P}=0.014)$. No correlation was noted between MUC1-Tn expression and other clinicopathological variables (Table II).

Correlation between MUC1-Tn expression and radiological features of tumors. Fig. 3A shows representative $\mathrm{CT}$ and ${ }^{18} \mathrm{~F}-\mathrm{FDG}-\mathrm{PET}$ scan images of lung ADC cases and MUC1-Tn staining of the corresponding resected specimens. A significantly higher $\mathrm{C} / \mathrm{T}$ ratio from the preoperative $\mathrm{CT}$ images was observed in tumors with MUC1-Tn-H (Fig. $3 \mathrm{~B}, \mathrm{P}=0.013)$. The $\mathrm{SUV}_{\max }$ was also higher in tumors with MUC1-Tn-H ( $\mathrm{P}<0.001$; Fig. 3C).

Correlation between MUC1-Tn expression and epithelial-mesenchymal transition (EMT). E-cadherin was detected in 97 of 171 lung ADC tumors (56.7\%), and Vimentin expression was positive in 68 tumors $(39.8 \%)$. According to the expression of E-cadherin and Vimentin, we classified tumors into the epithelial (76 cases [44.4\%]), intermediate (49 cases [28.7\%]), and mesenchymal (46 cases [26.9\%]) groups. Fig. 4A shows representative epithelial and mesenchymal tumors. The MUC1-Tn positivity of the mesenchymal group was higher than that of the epithelial or intermediate groups $(\mathrm{P}<0.01$ and $\mathrm{P}<0.01$, respectively; Fig. 4B).

Prognostic significance of MUC1-Tn expression. Analysis using the Kaplan-Meier method indicated significant associations between MUC1-Tn-H in lung ADCs and 5-year overall and disease-free survival $(\mathrm{P}=0.011$ and $\mathrm{P}<0.001$, respectively, by the log-rank test; Fig. 5). We also performed univariate analysis to evaluate associations between patient prognosis and other factors in patients with lung ADC. Advanced pT status $(\mathrm{P}<0.001)$, advanced $\mathrm{pN}$ status $(\mathrm{P}<0.001)$, advanced pleural invasion $(\mathrm{P}<0.001)$, high preoperative CEA levels $(\mathrm{P}=0.002)$, high $\mathrm{C} / \mathrm{T}$ ratio $(\mathrm{P}=0.002)$, high $\mathrm{SUV}_{\max }(\mathrm{P}<0.001)$, and MUC1-Tn positivity $(\mathrm{P}<0.001)$ were significantly associated with poor prognosis (Table II). MUC1-Tn expression was also identified as an independent prognostic factor in patients with ADC (hazard ratio (HR) 1.965, 95\% confidence intervals (CIs), 1.095-3.526, $\mathrm{P}=0.024)$, as were pT (HR 3.142, 95\% CIs, 1.635-6.035, $\mathrm{P}<0.001$ ) and $\mathrm{pN}(\mathrm{HR} 4.551,95 \% \mathrm{CIs}, 2.491-8.315, \mathrm{P}<0.001)$ status, in the $\mathrm{pT}$ and $\mathrm{pN}$ adjusted model by multivariate analysis (Table III). 
A

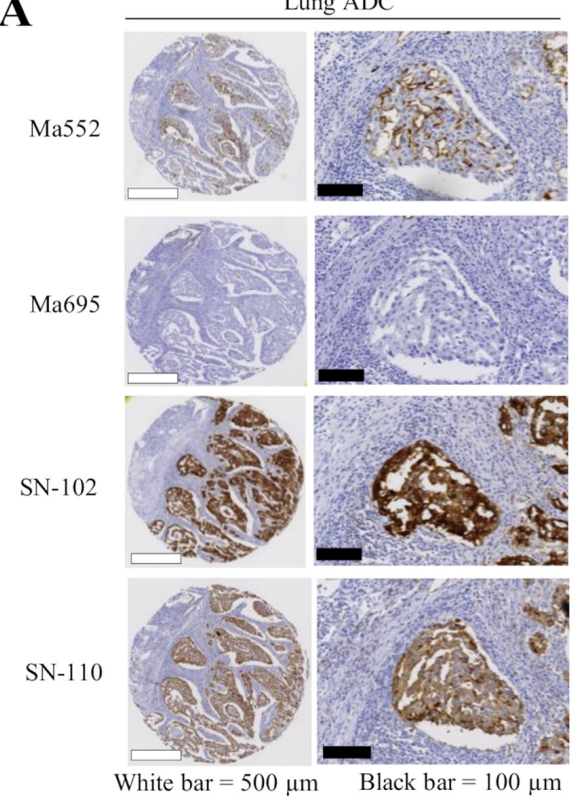

B

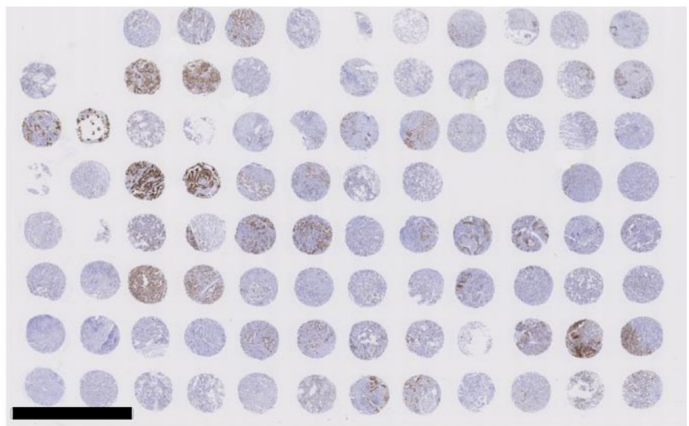

Black bar $=5 \mathrm{~mm}$

C

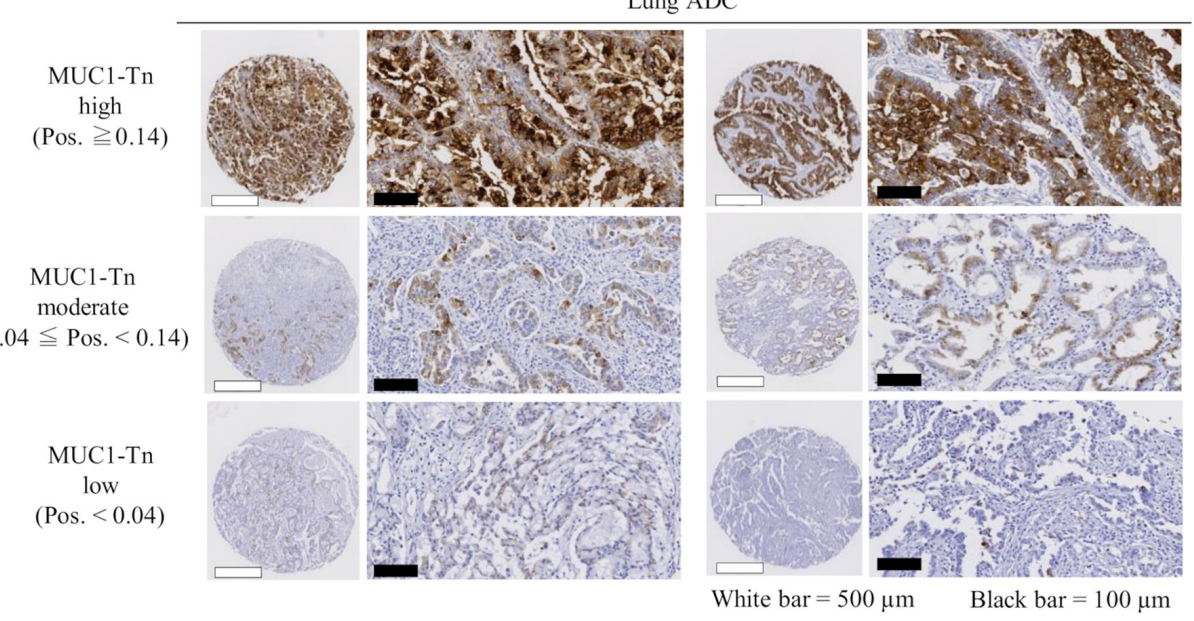

Figure 2. Expression levels of MUC1-Tn in lung cancer tissues. (A) MUC1-Tn exhibited stronger staining in tumor cells than commercially available MUC1 antibodies. White scale bars, $500 \mu \mathrm{m}$; black scale bars, $100 \mu \mathrm{m}$. (B) A representative overall view of a tissue microarray slide for MUC1-Tn staining. Black scale bar, $5 \mathrm{~mm}$. (C) Representative examples of MUC1-Tn protein expression in lung adenocarcinoma. High (top), moderate (middle) and low (bottom) expression levels of MUC1-Tn. White scale bars, $500 \mu \mathrm{m}$; black scale bars, $100 \mu \mathrm{m}$. ADC, adenocarcinoma; MUC1-Tn, mucin 1 Tn-antigen; Pos., positivity; MUC1, mucin 1.

\section{Discussion}

In the present study, we found that the MUC1-Tn epitope-defined antibody has high specificity for lung ADC cells and moderate or high MUC1-Tn expression was observed in three-quarters of lung ADCs. High MUC1-Tn is strongly associated with poor survival and the results presented herein have potential to open up novel therapeutic target for lung ADCs.

The specificity of antibodies for cancer cells is pivotal for antibody therapy. This is especially true in the treatment of lung cancer, as patients with other lung disorders such as interstitial pneumonia and emphysema sometimes have fatal consequences once respiratory side effects develop. In this study, a novel epitope-defined antibody that specifically recognizes MUC1 with cancer-associated carbohydrate antigens, O-glycans including Tn and sialyl-Tn, was developed by our original epitope-mapping analyses. Furthermore, screening normal lung and lung cancer tissues using TMAs, it was found that $\mathrm{SN}-102$, which recognizes the $\mathrm{Tn}$ antigen, had high specificity for cancer tissues, and there was no expression in normal lung tissue. Therefore, our results are completely different compared with those reported in previous studies regarding antibodies that simply recognize MUC1. Recently, genetically modified $\mathrm{T}$ cells expressing chimeric antigen receptors that recognized MUC1-Tn were found to have therapeutic efficacy in xenograft models of $\mathrm{T}$ cell leukemia and pancreatic cancer (19). Although further investigation is needed to validate this result, targeting cancer-associated carbohydrate antigens can be important for the treatment of lung cancer.

MUC1-Tn overexpression is common in multiple cancer types, such as gastric cancer $(20)$, colon cancer $(21,22)$ and breast cancer (23). Increased O-linked glycosylation of MUC1, including linkage of the Tn antigen and sialyl-Lewis-X, has 
Table II. Association between MUC1-Tn expression and clinicopathological features in patients with lung ADC.

\begin{tabular}{|c|c|c|c|}
\hline Variables & No. of cases & No. of cases with high MUC1-Tn expression, n (\%) & P-value ${ }^{b}$ \\
\hline All lung ADC cases & 175 & $44(25.1)$ & \\
\hline \multicolumn{4}{|l|}{ Age, years } \\
\hline$\leq 68$ & 92 & $22(23.9)$ & \multirow[t]{2}{*}{0.690} \\
\hline$>68$ & 83 & $22(26.2)$ & \\
\hline \multicolumn{4}{|l|}{ Sex } \\
\hline Male & 84 & $28(33.3)$ & \multirow[t]{2}{*}{$0.016^{\mathrm{d}}$} \\
\hline Female & 91 & $16(17.6)$ & \\
\hline \multicolumn{4}{|l|}{ Smoking status ${ }^{\mathrm{c}}$} \\
\hline Current or ex-smoker & 107 & $34(31.8)$ & \multirow[t]{3}{*}{$0.005^{\mathrm{d}}$} \\
\hline Non-smoker & 64 & $8(12.5)$ & \\
\hline NA & 4 & $2(50.0)$ & \\
\hline \multicolumn{4}{|l|}{ pT status ${ }^{\mathrm{a}}$} \\
\hline pT1a-c & 111 & $18(16.2)$ & \multirow[t]{2}{*}{$<0.001^{\mathrm{d}}$} \\
\hline pT2a-b+pT3+pT4 & 64 & $26(40.6)$ & \\
\hline \multicolumn{4}{|l|}{ pN status ${ }^{\mathrm{a}}$} \\
\hline pN0 & 144 & $34(23.6)$ & \multirow[t]{2}{*}{0.314} \\
\hline $\mathrm{pN} 1-2$ & 31 & $10(32.3)$ & \\
\hline \multicolumn{4}{|l|}{ Pleural invasion } \\
\hline Negative & 130 & $26(20.0)$ & \multirow[t]{2}{*}{$0.008^{d}$} \\
\hline Positive & 45 & $18(40.0)$ & \\
\hline \multicolumn{4}{|l|}{ Lymphatic invasion $^{\mathrm{c}}$} \\
\hline Negative & 29 & $14(48.3)$ & \multirow[t]{3}{*}{$<0.001^{\mathrm{d}}$} \\
\hline Positive & 64 & $9(14.1)$ & \\
\hline NA & 82 & $21(25.6)$ & \\
\hline \multicolumn{4}{|l|}{ Vascular invasion $^{\mathrm{c}}$} \\
\hline Negative & 27 & $12(44.4)$ & \multirow[t]{3}{*}{$0.005^{\mathrm{d}}$} \\
\hline Positive & 66 & $11(16.7)$ & \\
\hline NA & 82 & $21(25.6)$ & \\
\hline \multicolumn{4}{|c|}{ Pre-op Serum CEA level, ng/ml } \\
\hline$\leq 5.0$ & 108 & $20(18.5)$ & \multirow[t]{2}{*}{$0.010^{\mathrm{d}}$} \\
\hline$>5.0$ & 67 & $24(35.8)$ & \\
\hline \multicolumn{4}{|c|}{ Pre-op Serum CYFRA21-1 level ${ }^{\mathrm{c}}, \mathrm{ng} / \mathrm{ml}$} \\
\hline$\leq 2.1$ & 125 & $25(20.0)$ & \multirow[t]{3}{*}{$0.014^{\mathrm{d}}$} \\
\hline$>2.1$ & 44 & $17(38.6)$ & \\
\hline NA & 6 & $2(33.3)$ & \\
\hline
\end{tabular}

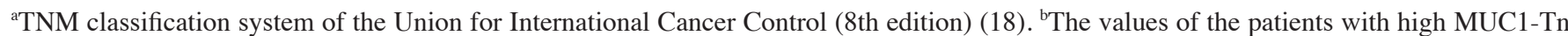
and the patients with moderate/low MUC1-Tn were compared using a $\chi^{2}$ test. ${ }^{\mathrm{T}}$ The values (except NA data) of the patients with high MUC1-Tn and the patients with moderate/low MUC1-Tn were compared using a $\chi^{2}$ test. ${ }^{\mathrm{d}} \mathrm{P}<0.05$. MUC1, mucin 1 ; ADC, adenocarcinoma; CEA, carcinoembryonic antigen; CYFRA21-1, cytokeratin 19 fragment; Pre-op, preoperative; NA, not available.

been also reported in lung cancer (24). O-glycans (Tn, sTn and $\mathrm{T}$ antigen) are synthesized in the Golgi apparatus using several glycosyltransferases (such as T-synthase and C1GALT1). The unique molecular chaperone of T-synthase is COSMC, which aids its folding in the endoplasmic reticulum. Abnormal expression of C1GALT1, somatic mutations, or hypermethylation of COSMC cause the dysregulation of O-glycans $(7,10,25)$. To confirm our results using clinical specimens, we first wanted to find MUC1-Tn-expressed cell lines to conduct an in vitro experiment. We performed immunohistochemical analyses using a MUC1-Tn monoclonal antibody SN-102 with cellblock materials of several lung cancer cell lines. However, we did not find any cell line with MUC1-Tn high expression (data not shown). To obtain a MUC1-Tn-expressed cell line, we will need to perform cell sorting for C1GALT1 or COSMC knockout cells $(9,10)$. 
Table III. Cox proportional hazards model analysis of prognostic factors in patients with adenocarcinoma $(n=175)$.

\begin{tabular}{|c|c|c|c|c|c|c|}
\hline \multirow[b]{2}{*}{ Variables } & \multicolumn{3}{|c|}{ Univariate analysis } & \multicolumn{3}{|c|}{ Multivariate analysis } \\
\hline & HR & $95 \% \mathrm{CI}$ & P-value & HR & $95 \% \mathrm{CI}$ & P-value \\
\hline Age ( $\leq 68$ vs. $>68$ years $)$ & 1.294 & $0.729-2.298$ & 0.397 & & & \\
\hline Sex (male vs. female) & 1.172 & $0.665-2.064$ & 0.583 & & & \\
\hline Brinkman index ( $\leq 400$ vs. $>400)$ & 1.239 & $0.699-2.196$ & 0.463 & & & \\
\hline pT (pT2-4 vs.pT1) & 5.255 & $2.843-9.715$ & $<0.001^{\mathrm{a}}$ & 3.142 & $1.635-6.035$ & $<0.001^{\mathrm{a}}$ \\
\hline pN (pN1-2 vs. pN0) & 7.013 & $3.936-12.495$ & $<0.001^{\mathrm{a}}$ & 4.551 & $2.491-8.315$ & $<0.001^{\mathrm{a}}$ \\
\hline Pleural invasion (positive vs. negative) & 3.523 & $1.933-6.226$ & $<0.001^{\mathrm{a}}$ & & & \\
\hline $\mathrm{CEA}(>5.0 \mathrm{vs} . \leq 5.0 \mathrm{ng} / \mathrm{ml})$ & 2.478 & $1.399-4.388$ & $0.002^{\mathrm{a}}$ & & & \\
\hline CYRFA21-1 (>2.1 vs. $\leq 2.1 \mathrm{ng} / \mathrm{ml})$ & 1.600 & $0.878-2.916$ & 0.125 & & & \\
\hline $\mathrm{C} / \mathrm{T}$ ratio $(\leq 0.5$ vs. $>0.5)$ & 9.687 & $2.348-39.955$ & $0.002^{\mathrm{a}}$ & & & \\
\hline FDG-PET SUV $_{\max }(>2.4$ vs. $\leq 2.4)$ & 4.338 & $2.202-8.546$ & $<0.001^{\mathrm{a}}$ & & & \\
\hline MUC1-Tn expression (high vs. moderate/low) & 2.797 & $1.579-4.956$ & $<0.001^{\mathrm{a}}$ & 1.965 & $1.095-3.526$ & $0.024^{\mathrm{a}}$ \\
\hline
\end{tabular}

${ }^{a} \mathrm{P}<0.05$. CI, confidence interval; CEA, carcinoembryonic antigen; CYRFA21-1, cytokeratin 19 fragment; C/T ratio, consolidation/tumor ratio; FDG-PET $\mathrm{SUV}_{\max },{ }^{18} \mathrm{~F}$-fluorodeoxyglucose positron emission tomography maximum standardized uptake value; HR, hazard ratio; MUC1-Tn, mucin 1 Tn-antigen.

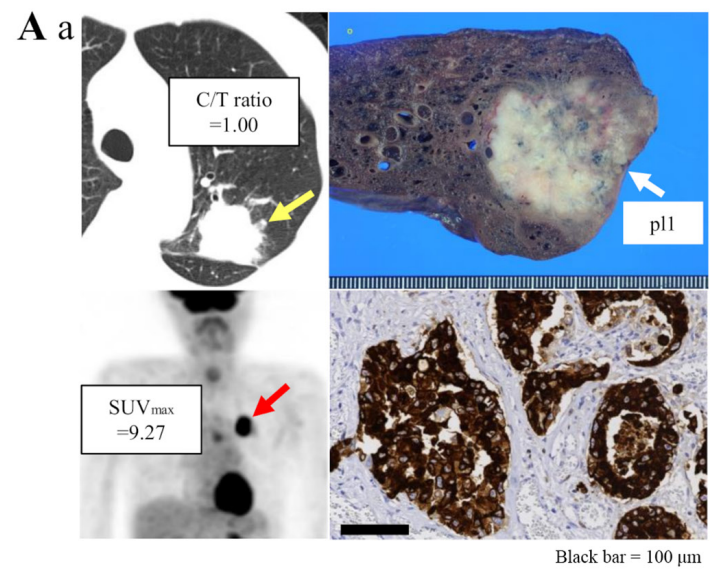

B

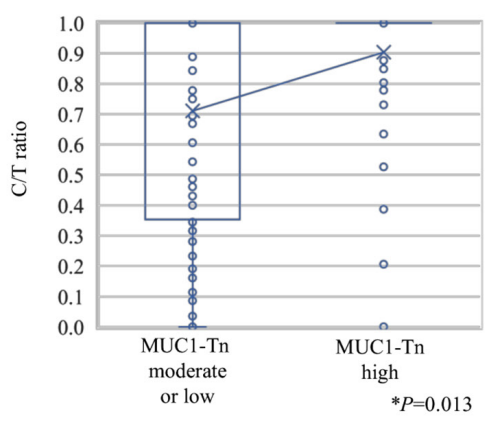

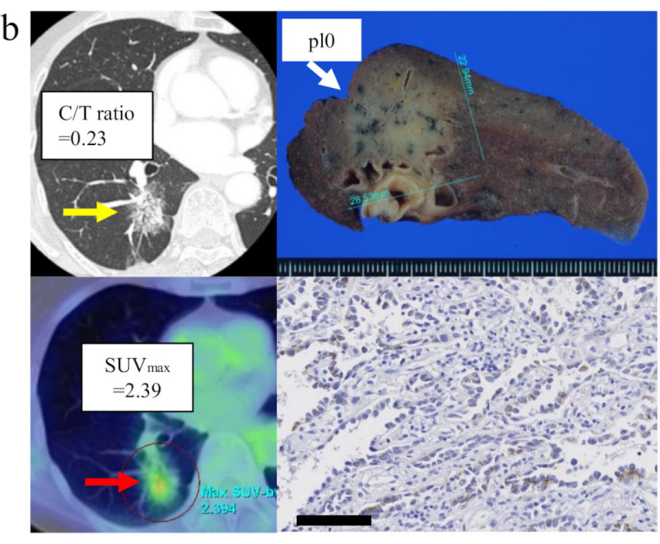

C

C

***

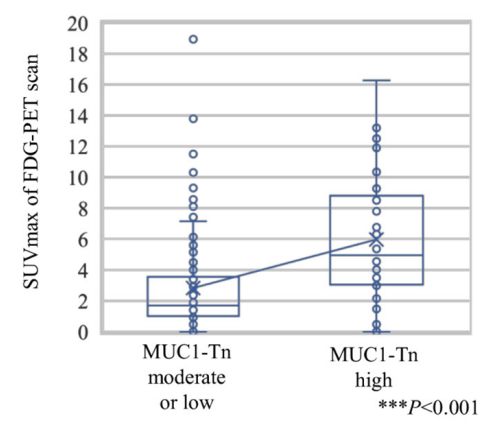

Figure 3. Association between radiological features and MUC1-Tn expression in lung adenocarcinoma. (A) Representative CT and FDG-PET scan images according to MUC1-Tn expression. (Aa) A case with high expression levels of MUC1-Tn. (Ab) A case with low expression levels of MUC1-Tn. Scale bars, $100 \mu \mathrm{m}$. (B) Association between the $\mathrm{C} / \mathrm{T}$ ratio and MUC1-Tn expression. ${ }^{*} \mathrm{P}<0.05$. (C) Association between SUV ${ }_{\text {max }}$ of FDG-PET scan and MUC1-Tn expression. For the box-and-whisker plot, the ends of the box are the upper and lower quartiles, and the median is marked by a vertical line inside the box. The whiskers are two lines outside the box that extend to the highest and lowest values without outliers, which are $\geq 1.5$ times the interquartile range. The crosses represent the mean. ${ }^{* * *} \mathrm{P}<0.001$. MUC1-Tn, mucin 1 Tn-antigen; FDG-PET, ${ }^{18} \mathrm{~F}$-fluorodeoxyglucose positron emission tomography; pl0, tumor with no pleural involvement beyond its elastic layer; pl1, tumor that has invaded beyond the elastic layer of the visceral pleura but is not exposed on the pleural surface; $\mathrm{C} / \mathrm{T}$ ratio, consolidation/tumor ratio; $\mathrm{SUV}_{\max }$, maximum standardized uptake value.

Although the role of Tn antigen in the development of cancer is still unclear, several studies have reported the underlying mechanisms of the relationship between MUC1-Tn and poorer prognosis. The knockout of C1GALT1 enhanced the growth 

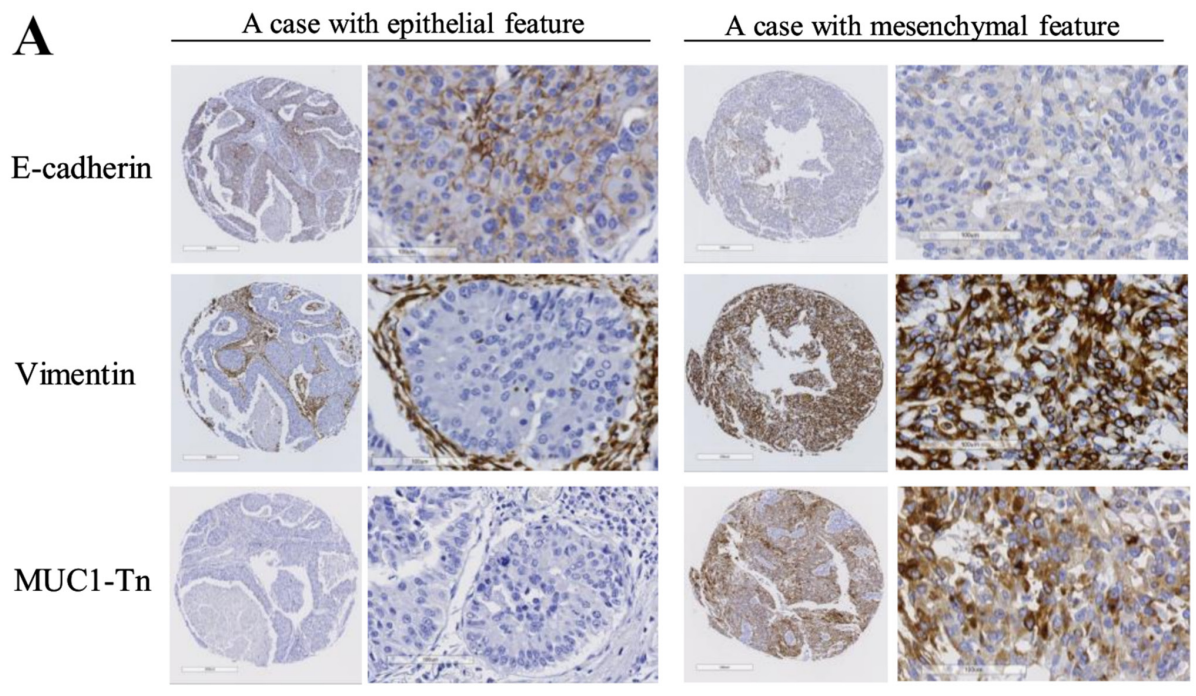

B

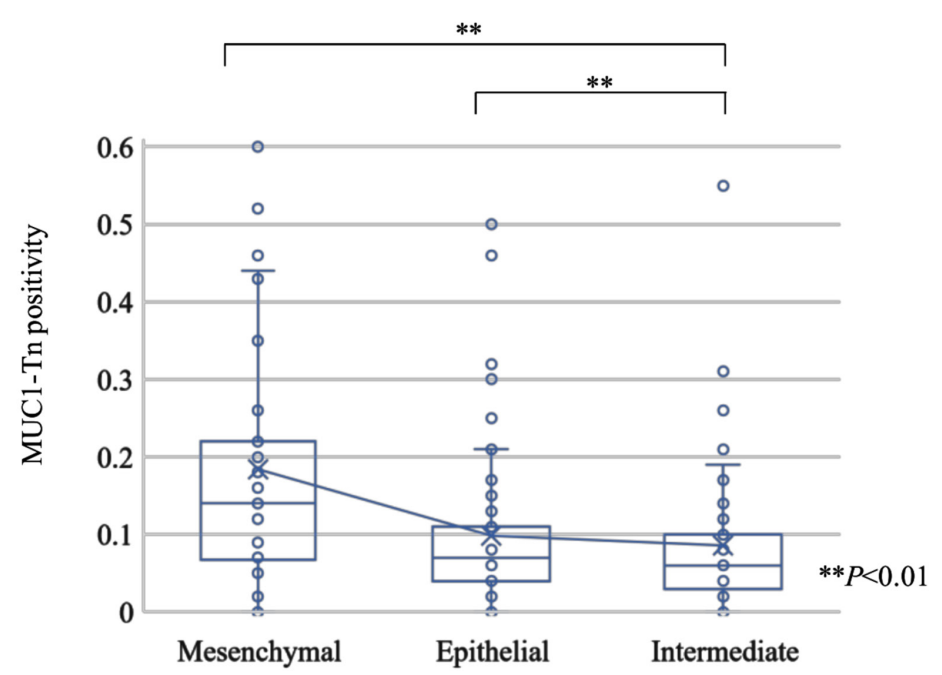

Figure 4. Association between features of EMT and MUC1-Tn expression in lung adenocarcinoma. (A) Immunohistochemical staining of E-cadherin and Vimentin, and MUC1-Tn expression in representative epithelial and mesenchymal tumors. (B) Association between EMT status and MUC1-Tn expression. Cases with mesenchymal features ( 46 cases; $26.9 \%$ ), epithelial features (76 cases; $44.4 \%)$ and intermediate features (49 cases; 28.7\%). ${ }^{* *} \mathrm{P}<0.01$. EMT, epithelial-mesenchymal transition; MUC1-Tn, mucin 1 Tn-antigen.
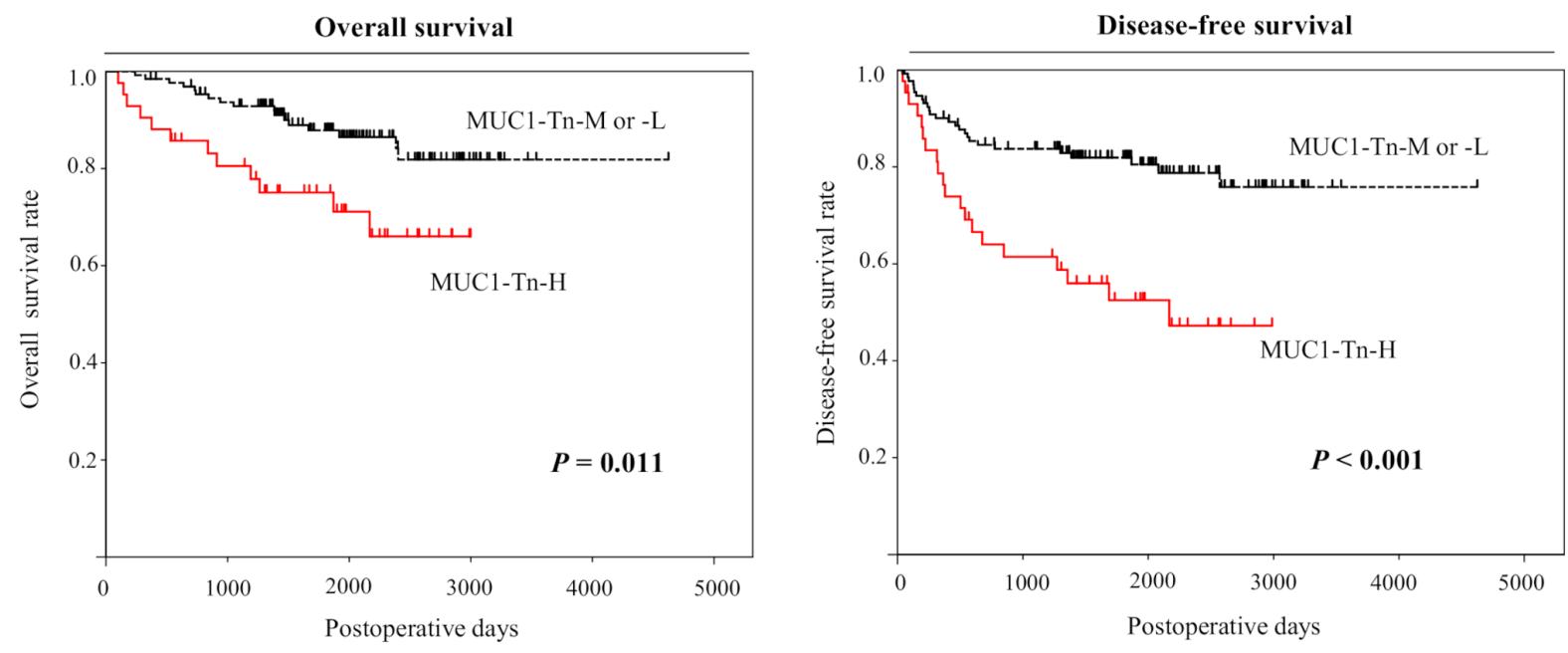

Figure 5. Kaplan-Meier analysis of overall and disease-free survival in patients with lung adenocarcinoma according to MUC1-Tn expression. The 5-year overall survival rate was $87.9 \%$ for patients with MUC1-Tn-M or $-\mathrm{L}(\mathrm{n}=131)$ and $69.3 \%$ for patients with MUC1-Tn-H $(\mathrm{n}=44$; $\mathrm{P}=0.011)$. The 5-year disease-free survival rates were $79.6 \%$ for patients with MUC1-Tn-M or -L and $46.1 \%$ for patients with MUC1-Tn-H ( $\mathrm{P}<0.001)$. MUC1-Tn, mucin 1 Tn-antigen; M, moderate; L, low; $\mathrm{H}$, high. 
and migration of pancreatic cancer cells (9). Additionally, COSMC knockout cells expressing truncated O-glycans (such as Tn and/or STn) promote cell proliferation, prevent differentiation, enhance invasive and migratory properties, and impair cell-cell adhesion in culture (10). Aberrant forms or amounts of O-glycans are also thought to provide ligands that interact with growth factors, lectins, selectins, and cell adhesion molecules in cancer cells. The dense layers of O-glycans may also help control the local microenvironment and protect cancer cells from adverse growth conditions during invasion and metastasis (26). This study showed that MUC1-Tn overexpression correlates with tumor extension and pleural invasion. Besides, the binding between Tn antigen and macrophage galactose-type C-type lectin (MGL) in situ, which is expressed in dendritic cells and macrophages, may have immunosuppressive effects and may enable the tumor escape immunosurveillance (27-29). High expression of mucin and altered glycosylation are related to the expression of galectin-3, which can bind to Tn antigen, contributing to metastasis and escape from immunosurveillance (30). This evasion of immune surveillance may be correlated with poor prognosis. A previous study showed that stage I lung ADCs with EMT conversion show solid-dominant nodules on CT that are not visible in ADCs without conversion, and the $\mathrm{SUV}_{\text {max }}$ is higher in the mesenchymal group than in the epithelial group (31). Our study also showed that tumors with high expression of MUC1-Tn had higher $\mathrm{SUV}_{\max }$ on FDG-PET scans, suggesting MUC1-Tn overexpression also represents a more malignant feature of lung cancer as per radiological examinations. Hypoxic stress causes a shift from aerobic oxidative phosphorylation to anaerobic glycolysis, with high rates of glucose and glutamine uptake (32). In this context, adaptation to hypoxia and cellular energetic reprogramming occurs mostly in a hypoxia-inducible factor-1 (HIF-1) alpha-dependent manner and is frequently accompanied by cell dedifferentiation and acquisition of mesenchymal features (33). Expression and/or activity levels of glucose transporters contribute to the pattern and intensity of ${ }^{18} \mathrm{~F}$-FDG. MUC1 is also directly regulated by HIF- $1 \alpha$ and affects the invasive and migratory properties of cancer cells (34). Depolarized MUC1 expression was significantly associated with the expression of glucose transporters-1 (GLUT1) and poor outcomes in lung cancer (35). It is expected that the relationship between MUC1-Tn expression and glucose transporters will be examined in future.

Aberrant glycosylation of MUC1-N in response to cigarette smoke initiates EMT by degrading E-cadherin and damages cell-cell adhesions, resulting in changes in cellular polarity. The cells become spindle-shaped and motile, an early indicator of malignant transformation and an invasive nature $(6,36)$. Changes in mucin localization due to loss of epithelial cell polarity are also associated with malignant alteration (37). In this study, we proved that high levels of MUC1-Tn expression were correlated with smoking habits, low E-cadherin, and high Vimentin, suggesting mesenchymal features.

This study has some limitations. We determined the EMT status solely via E-cadherin and Vimentin expression. This may need further validation using a panel of EMT markers such as Snail and Twist. Analyses of morphological characteristics or phenotypes of EMT should be considered. Additionally, some cases had no MUC1-Tn expression even after EMT; therefore, further investigation, including expression of other O-glycans, may be needed.

In conclusion, moderate to high levels of MUC1-Tn expression were observed in three-quarters of primary lung ADC patients, and normal lung tissue had no expression of MUC-Tn. Overexpression of MUC1-Tn was strongly associated with EMT potential and poor survival in patients with lung ADC. These results strongly suggest that MUC1-Tn can be a valuable marker in patients who are likely to show poor prognosis and can be used to develop improved antibody immunotherapeutics for lung ADC.

\section{Acknowledgements}

Not applicable.

\section{Funding}

No funding was received.

\section{Availability of data and materials}

All data generated or analyzed during this study are included in this published article.

\section{Authors' contributions}

TK performed the experimental design, most of the experiments and analyses, drafted the manuscript, and was involved in the conception and design of the study. HU contributed to the preparation and the review of the tissue microarray. $\mathrm{KCH}$ participated in the planning/design of the tissue microarray experiments and supervised pathological review. TK, $\mathrm{HU}$ and $\mathrm{KCH}$ assessed all raw data and confirmed the authenticity. YH was also involved in collecting clinical tissue samples and accessing clinical databases. AN, AO and KT conducted some supporting immunohistochemical experiments. KN and MS were involved in the production of MUC1-Tn ED antibody and in acquisition of data regarding immunogen and the chemical formula for the antibody. KK, SW, YM and YH supervised the study and were involved in the conception and design of the study, and proofread the manuscript. All authors read and approved the final manuscript.

\section{Ethics approval and consent to participate}

The protocol was approved by the appropriate Institutional Review Board of Hokkaido University Hospital (approval no. \#015-0367; Sapporo, Japan). Each patient provided written informed consent at the time of surgery.

\section{Patient consent for publication}

Not applicable.

\section{Competing interests}

KN and MS are affiliated with Medicinal Chemistry Pharmaceuticals, Co., Ltd., who made the antibody that the study is largely based on. The other authors declare that they have no competing interests. 


\section{References}

1. National Cancer Institute: Surveillance, Epidemiology, and End Results Program. Accessed from: https://seer.cancer.gov/ statfacts/html/common.html2020.

2. Nath S and Mukherjee P: MUC1: A multifaceted oncoprotein with a key role in cancer progression. Trends Mol Med 20: 332-342, 2014.

3. Parry S,SilvermanHS,McDermottK, Willis A,HollingsworthMA and Harris A: Identification of MUC1 proteolytic cleavage sites in vivo. Biochem Biophys Res Commun 283: 715-720, 2001.

4. Siddiqui J, Abe M, Hayes D, Shani E, Yunis E and Kufe D: Isolation and sequencing of a cDNA coding for the human DF3 breast carcinoma-associated antigen. Proc Natl Acad Sci USA 85: 2320-2323, 1988.

5. Gendler S, Taylor-Papadimitriou J, Duhig T, Rothbard J and Burchell J: A highly immunogenic region of a human polymorphic epithelial mucin expressed by carcinomas is made up of tandem repeats. J Biol Chem 263: 12820-12823, 1988.

6. Qu J, Yu H, Li F, Zhang C, Trad A, Brooks C, Zhang B, Gong T, Guo Z, Li Y, et al: Molecular basis of antibody binding to mucin glycopeptides in lung cancer. Int J Oncol 48: 587-594, 2016.

7. Fu C, Zhao H, Wang Y, Cai H, Xiao Y, Zeng Y and Chen $\mathrm{H}$ Tumor-associated antigens: Tn antigen, sTn antigen, and T antigen. HLA 88: 275-286, 2016.

8. Ju T and Cummings RD: A unique molecular chaperone Cosmc required for activity of the mammalian core 1 beta 3-galactosyltransferase. Proc Natl Acad Sci USA 99: 16613-16618, 2002.

9. Chugh S, Barkeer S, Rachagani S, Nimmakayala RK, Perumal N, Pothuraju R, Atri P, Mahapatra S, Thapa I, Talmon GA, et al: Disruption of C1galt1 gene promotes development and metastasis of pancreatic adenocarcinomas in mice. Gastroenterology 155: $1608-1624,2018$

10. Radhakrishnan P, Dabelsteen S, Madsen FB, Francavilla C, Kopp KL, Steentoft C, Vakhrushev SY, Olsen JV, Hansen L, Bennett EP, et al: Immature truncated O-glycophenotype of cancer directly induces oncogenic features. Proc Natl Acad Sci USA 111: E4066-E4075, 2014.

11. Guddo F, Giatromanolaki A, Koukourakis MI, Reina C, Vignola AM, Chlouverakis G, Hilkens J, Gatter KC, Harris AL and Bonsignore G: MUC1 (episialin) expression in non-small cell lung cancer is independent of EGFR and c-erbB-2 expression and correlates with poor survival in node positive patients. J Clin Pathol 51: 667-671, 1998.

12. Nagai S, Takenaka K, Sonobe M, Ogawa E, Wada H and Tanaka F: A novel classification of MUC1 expression is correlated with tumor differentiation and postoperative prognosis in non-small cell lung cancer. J Thorac Oncol 1: 46-51, 2006.

13. Kuemmel A, Single K, Bittinger F, Faldum A, Schmidt LH, Sebastian M, Micke P, Taube C, Buhl R and Wiewrodt R: TA-MUC1 epitope in non-small cell lung cancer. Lung Cancer 63: 98-105, 2009

14. Ishikawa N, Hattori N, Yokoyama A and Kohno N: Utility of KL-6/MUC1 in the clinical management of interstitial lung diseases. Respir Investig 50: 3-13, 2012.

15. Tanaka S, Hattori N, Ishikawa N, Shoda H, Takano A, Nishino R, Okada M, Arihiro K, Inai K, Hamada H, et al: Krebs von den Lungen-6 (KL-6) is a prognostic biomarker in patients with surgically resected nonsmall cell lung cancer. Int J Cancer 130: 377-387, 2012

16. Naito S, Takahashi T, Onoda J, Uemura S, Ohyabu N, Takemoto H, Yamane S, Fujii I, Nishimura SI and Numata Y: Generation of novel anti-MUC1 monoclonal antibodies with designed carbohydrate specificities using MUC1 glycopeptide library. ACS Omega 2: 7493-7505, 2017.

17. Travis W, Brambilla E, Burke AP, Marx A and Nicholson AG: WHO Classification of Tumours of the Lung, Pleura, Thymus and Heart. International Agency for Research on Cancer, Lyon, 2015.

18. Brierley JD, Gospodarowicz MK and Wittekind C (eds): Union for International Cancer Control (UICC): TNM Classification of Malignant Tumours. 8th edition. Wiley-Blackwell, Hoboken, NJ, p272, 2017.
19. Posey AD Jr, Schwab RD, Boesteanu AC, Steentoft C, Mandel U, Engels B, Stone JD, Madsen TD, Schreiber K, Haines KM, et al: Engineered CAR-T cells targeting the cancer-associated Tn-glycoform of the membrane mucin MUC1 control adenocarcinoma. Immunity 44: 1444-1454, 2016.

20. Li T, Mo C, Qin X, Li S, Liu Y and Liu Z: Glycoprofiling of early gastric cancer using lectin microarray technology. Clin Lab 64 : 153-161, 2018.

21. Konno A, Hoshino Y, Terashima S, Motoki R and Kawaguchi T: Carbohydrate expression profile of colorectal cancer cells is relevant to metastatic pattern and prognosis. Clin Exp Metastasis 19: 61-70, 2002.

22. Byrd JC and Bresalier RS: Mucins and mucin binding proteins in colorectal cancer. Cancer Metastasis Rev 23: 77-99, 2004.

23. Welinder C, Baldetorp B, Blixt O, Grabau D and Jansson B: Primary breast cancer tumours contain high amounts of IgA1 immunoglobulin: An immunohistochemical analysis of a possible carrier of the tumour-associated Tn antigen. PLoS One 8: e61749, 2013.

24. Pinto R, Carvalho AS, Conze T, Magalhães A, Picco G, Burchell JM, Taylor-Papadimitriou J, Reis CA, Almeida R, Mandel U, et al: Identification of new cancer biomarkers based on aberrant mucin glycoforms by in situ proximity ligation. J Cell Mol Med 16: 1474-1484, 2012.

25. Ju T, Lanneau GS, Gautam T, Wang Y, Xia B, Stowell SR, Willard MT, Wang W, Xia JY, Zuna RE, et al: Human tumor antigens Tn and sialyl Tn arise from mutations in Cosmc. Cancer Res 68: 1636-1646, 2008.

26. Häuselmann I and Borsig L: Altered tumor-cell glycosylation promotes metastasis. Front Oncol 4: 28, 2014.

27. Higashi N, Fujioka K, Denda-Nagai K, Hashimoto S, Nagai S, Sato T, Fujita Y, Morikawa A, Tsuiji M, Miyata-Takeuchi M, et al: The macrophage C-type lectin specific for galactose/ $\mathrm{N}$-acetylgalactosamine is an endocytic receptor expressed on monocyte-derived immature dendritic cells. J Biol Chem 277: 20686-20693, 2002.

28. Ju T, Wang Y, Aryal RP, Lehoux SD, Ding X, Kudelka MR, Cutler C, Zeng J, Wang J, Sun X, et al: Tn and sialyl-Tn antigens, aberrant O-glycomics as human disease markers. Proteomics Clin Appl 7: 618-631, 2013.

29. Napoletano C, Rughetti A, Agervig Tarp MP, Coleman J, Bennett EP, Picco G, Sale P, Denda-Nagai K, Irimura T, Mandel U, et al: Tumor-associated Tn-MUC1 glycoform is internalized through the macrophage galactose-type C-type lectin and delivered to the HLA class I and II compartments in dendritic cells. Cancer Res 67: 8358-8367, 2007.

30. Kölbl AC, Jeschke U, Friese K and Andergassen U: The role of TF- and Tn-antigens in breast cancer metastasis. Histol Histopathol 31: 613-621, 2016.

31. Matsubara T, Tagawa T, Takada K, Toyokawa G, Shimokawa M, Kozuma Y, Akamine T, Haro A, Osoegawa A and Mori M: Clinical and prognostic significance of the epithelial-mesenchymal transition in stage IA lung adenocarcinoma: A propensity score-matched analysis. Clin Lung Cancer 20: e504-e513, 2019.

32. Warburg O: On the origin of cancer cells. Science 123: 309-314, 1956.

33. Peixoto A, Fernandes E, Gaiteiro C, Lima L, Azevedo R, Soares J, Cotton S, Parreira B, Neves M, Amaro T, et al: Hypoxia enhances the malignant nature of bladder cancer cells and concomitantly antagonizes protein $\mathrm{O}$-glycosylation extension. Oncotarget 7: 63138-63157, 2016

34. Aubert S, Fauquette V, Hémon B, Lepoivre R, Briez N, Bernard D, Van Seuningen I, Leroy X and Perrais M: MUC1, a new hypoxia inducible factor target gene, is an actor in clear renal cell carcinoma tumor progression. Cancer Res 69: 5707-5715, 2009.

35. Kaira K, Okumura T, Nakagawa K, Ohde Y, Takahashi T, Murakami H, Naito T, Endo M, Kondo H, Nakajima T, et al: MUC1 expression in pulmonary metastatic tumors: A comparison of primary lung cancer. Pathol Oncol Res 18: 439-447, 2012.

36. Hollingsworth MA and Swanson BJ: Mucins in cancer: Protection and control of the cell surface. Nat Rev Cancer 4: 45-60, 2004.

37. Kufe DW: Mucins in cancer: Function, prognosis and therapy. Nat Rev Cancer 9: 874-885, 2009. 